\title{
Activation of secondary metabolite-biosynthetic gene clusters by generating rsmG mutations in Streptomyces griseus
}

\author{
Yukinori Tanaka ${ }^{1,2}$, Shinji Tokuyama ${ }^{2}$ and Kozo Ochi ${ }^{1}$ \\ Unlike other Streptomyces spp., the streptomycin producer Streptomyces griseus IF013189 shows emergence of a small \\ fraction of $r s m G$ and $r p s L$ mutants among spontaneous low- or high-level streptomycin-resistant mutants. rsmG, but not $r p s L$, \\ mutants showed greater ability (two- to threefold) to produce streptomycin, accompanied by enhanced transcription of metK \\ and $s t r R$, together with streptomycin biosynthetic genes, such as strB1, strD and strF, thus underlying the observed increase \\ in streptomycin production in the rsmG mutants. Moreover, $r s m G$ mutation was effective for activating the 'silent' or poorly \\ expressed secondary metabolite-biosynthetic genes present in S. griseus.
}

The Journal of Antibiotics (2009) 62, 669-673; doi:10.1038/ja.2009.97; published online 9 October 2009

Keywords: rsmG; silent genes; Streptomyces griseus; streptomycin resistance

\section{INTRODUCTION}

Actinomycetes produce a variety of natural products that are of major importance in the pharmaceutical industry. More than $50 \%$ of all antiinfective and anticancer compounds developed over the past 25 years have been natural products or derivatives thereof. ${ }^{1}$ In the past several years, the complete genome sequences of many organisms have been reported. The information from these genome projects indicated that Streptomyces coelicolor, S. avermitilis and S. griseus have 20, 25 and 34 clusters of genes involved in secondary metabolism, respectively, but only a few secondary metabolites are known in each case..$^{2-4}$ Recently, we described a practical method for increasing antibiotic production in bacteria by modulating ribosomal components (ribosomal proteins or rRNA), specifically by generating mutations conferring drug resistance, such as streptomycin resistance. ${ }^{5-7}$ This approach, called 'ribosome engineering, ${ }^{8}$ has several advantages including the ability to screen for drug resistance mutations by simple selection on drug-containing plates, even if the mutation frequency is extremely low (for example, $<10^{-10}$ ), and has been shown to be effective for improving the industrial strains, which had been bred to produce large amount of antibiotics. ${ }^{9} 10$ S. griseus is a filamentous, soil-living, Gram-positive bacteria, which produces an aminoglycoside antibiotic, streptomycin, and is characterized by the presence of a streptomycin self-resistance gene, $a p h D$, which encodes streptomycin-6-phosphotransferase. Here, we showed that $r s m G$ and $r p s L$ (conferring low and high levels of resistance to streptomycin, respectively) mutations can be generated in $S$. griseus, although this organism already possesses a relatively high level of self-resistance to this antibiotic produced by the organism itself. The effects of $r s m G$ and $r p s L$ mutations on streptomycin production were examined, along with those of many other 'silent' secondary metabolite-biosynthetic genes present in this organism.

\section{MATERIALS AND METHODS}

Bacterial strains and culture conditions

Streptomyces griseus IFO13189, a prototrophic streptomycin-producing wildtype strain, was used as the parental strain. Spontaneous streptomycin-resistant mutants were obtained as colonies that grew within 5-7 days after spores were spread on glucose-yeast extract-malt extract (GYM) agar ${ }^{11}$ containing various concentrations of streptomycin. Mutations in $r p s L$ or $r s m G$ genes were determined by DNA sequencing using the primers listed in Table 1. Cultivation was performed at $30^{\circ} \mathrm{C}$ (for preculture) or $25^{\circ} \mathrm{C}$ (for main culture) using SPY medium $^{11}$ with rotary shaking at 220 r.p.m.

Assay for streptomycin and determination of minimum inhibitory concentrations

Streptomycin was determined by bioassay using Bacillus subtilis ATCC6633 as the test organism. Minimum inhibitory concentrations (MICs) were determined by spotting spore solutions $\left(\sim 10^{6}\right)$ onto streptomycin-containing GYM plates, followed by incubation for 5 days at $30^{\circ} \mathrm{C}$.

\section{Total RNA preparation}

Total cellular RNA was prepared using Isogen reagent (Nippon Gene, Toyama, Japan). Each pellet was resuspended in $1 \mathrm{ml}$ of Isogen reagent and incubated at $50^{\circ} \mathrm{C}$ for $10 \mathrm{~min}$. After cooling, $0.2 \mathrm{ml}$ of chloroform was added. Each sample was mixed well by vortexing and centrifuged at $16000 \times g$ for $10 \mathrm{~min}$, and $0.6 \mathrm{ml}$ of the aqueous phase (top layer) was transferred to a clean tube. To each test tube, $0.4 \mathrm{ml}$ of chloroform was added and each sample was mixed by repeated inversions and centrifuged at $16000 \times \mathrm{g}$ for $10 \mathrm{~min}$. A portion $(0.6 \mathrm{ml})$ of the aqueous phase (top layer) was transferred to a clean tube. The RNA in each

${ }^{1}$ National Food Research Institute, Tsukuba, Ibaraki, Japan and ${ }^{2}$ Faculty of Agriculture, Department of Applied Biological Chemistry, Shizuoka University, Shizuoka, Japan Correspondence: Dr K Ochi, National Food Research Institute, 2-1-12 Kannondai, Tsukuba, Ibaraki 305-8642, Japan. E-mail: kochi@affrc.go.jp

Received 14 July 2009; revised 10 September 2009; accepted 18 September 2009; published online 9 October 2009 
Table 1 The primers used in this study

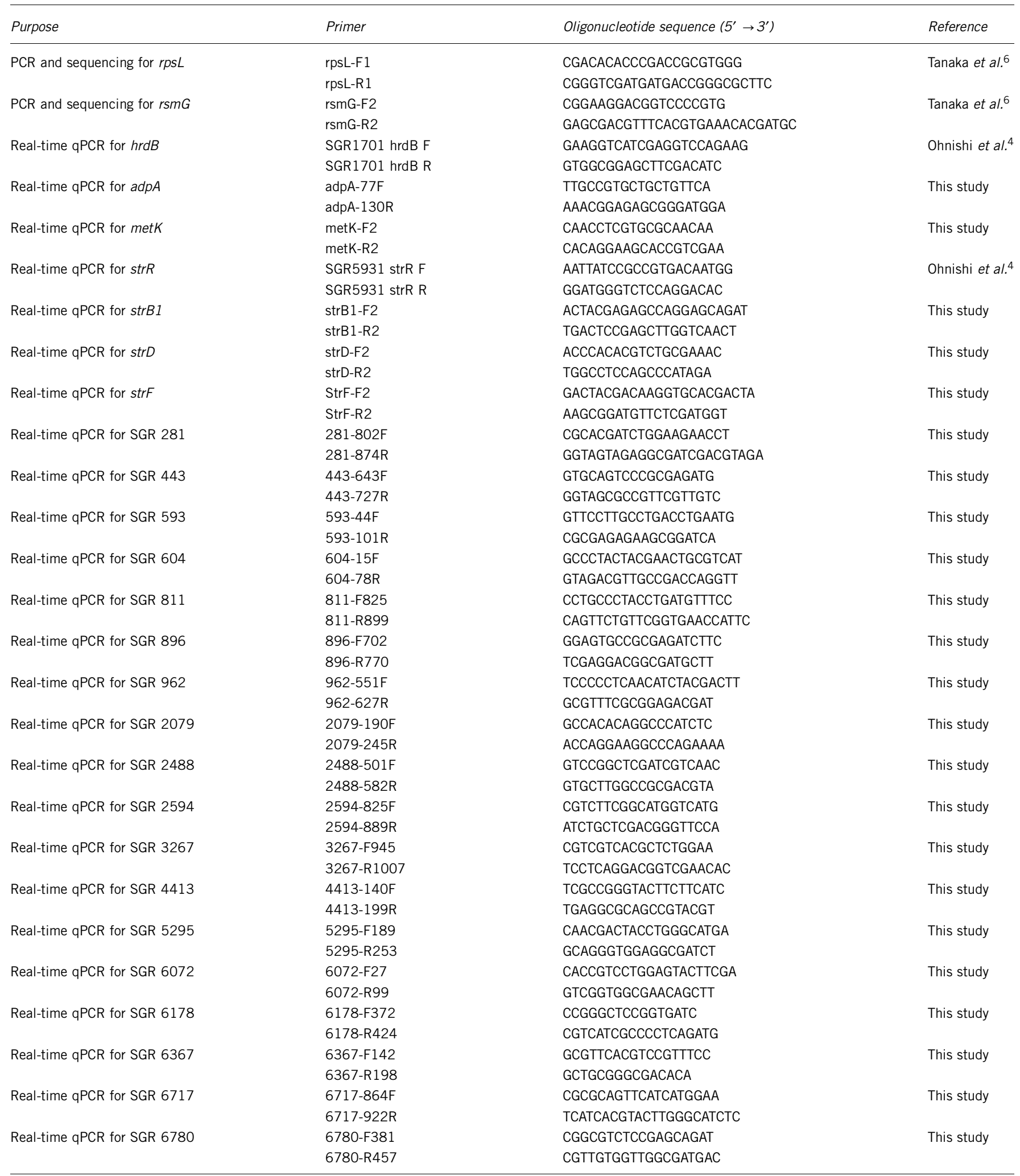

Abbreviation: qPCR, quantitative PCR.

tube was precipitated with an equal volume of isopropanol, and rinsed with $70 \%$ ethanol. The Isogen reagent treatment was repeated once more to prepare pure RNA and finally suspended with an appropriate volume of diethylpyrocarbonate-treated water.
Transcriptional analysis by real-time quantitative PCR

The total RNAs were prepared as described above. Contaminating DNA was removed by incubating each total RNA sample $(1 \mu \mathrm{g})$ with $1 \mathrm{U}$ of DNAse I (Invitrogen, Carlsbad, CA, USA) for $15 \mathrm{~min}$ at $25^{\circ} \mathrm{C}$. Reverse transcription 
Table 2 Characterization of streptomycin resistance mutations of Streptomyces griseus

\begin{tabular}{|c|c|c|c|c|c|c|c|}
\hline \multirow{2}{*}{$\begin{array}{l}\text { Streptomycin } \\
\text { concentration }\left(\mu \mathrm{g} \mathrm{m}^{-1}\right) \\
\text { used for selection }\end{array}$} & \multicolumn{2}{|c|}{ Mutation in ${ }^{\mathrm{a}}$} & \multirow{2}{*}{$\begin{array}{l}\text { Amino acid } \\
\text { substitution }\end{array}$} & \multirow{2}{*}{$\begin{array}{c}\text { Frequency of } \\
\text { mutants with } \\
\text { the same mutation }\end{array}$} & \multirow{2}{*}{$\begin{array}{l}\text { Minimum inhibitory } \\
\text { concentration to } \\
\text { streptomycin }\left(\mu \mathrm{g} \mathrm{ml^{-1 }}\right)^{\mathrm{b}}\end{array}$} & \multirow{2}{*}{$\begin{array}{c}\text { Streptomycin } \\
\text { productivity }\left(\mu g \mathrm{~m}^{-1}\right)^{\mathrm{c}}\end{array}$} & \multirow{2}{*}{$\begin{array}{l}\text { Designation } \\
\text { of mutant }\end{array}$} \\
\hline & $r s m G$ & $r p s L$ & & & & & \\
\hline \multirow[t]{9}{*}{70} & $\Delta 73 \mathrm{G}-76 \mathrm{~T}$ & & Frameshift & $1 / 86$ & 300 & $118 \pm 27$ & KO-1057 \\
\hline & $\begin{array}{l}\text { Insertion of } \\
\text { AGCTC at } 88\end{array}$ & & Frameshift & $1 / 86$ & 300 & $117 \pm 27$ & KO-1058 \\
\hline & $\begin{array}{l}\text { Insertion of } \\
\text { CCA at } 277\end{array}$ & & $\begin{array}{l}\text { Insertion of } \\
\text { Pro at } 93\end{array}$ & $1 / 86$ & 300 & $124 \pm 17$ & KO-1061 \\
\hline & $280 G \rightarrow C$ & & Ala94 $\rightarrow$ Pro & $1 / 86$ & 300 & $144 \pm 18$ & Ko-1062 \\
\hline & $331 \mathrm{C} \rightarrow \mathrm{T}$ & & $\operatorname{Arg} 111 \rightarrow \operatorname{Trp}$ & $1 / 86$ & 300 & $124 \pm 17$ & KO-1063 \\
\hline & $332 \mathrm{G} \rightarrow \mathrm{A}$ & & $\operatorname{Arg} 111 \rightarrow G \ln$ & $1 / 86$ & 300 & $130 \pm 5$ & KO-1064 \\
\hline & $\Delta 389 \mathrm{~T}-398 \mathrm{G}$ & & Frameshift & $1 / 86$ & 300 & $139 \pm 33$ & KO-1065 \\
\hline & $\Delta 623 \mathrm{~T}$ & & Frameshift & $1 / 86$ & 300 & $125 \pm 4$ & KO-1070 \\
\hline & $N D^{e}$ & & ND & $72 / 86$ & $-^{f}$ & $-^{f}$ & \\
\hline \multirow[t]{3}{*}{100} & $\Delta 260 G-291 G$ & & Frameshift & $1 / 30$ & 300 & $140 \pm 8$ & KO-1050 \\
\hline & $\Delta 389 \mathrm{~T}-398 \mathrm{G}$ & & Frameshift & $1 / 30$ & 300 & $162 \pm 17$ & Ko-1051 \\
\hline & ND & & ND & $28 / 30$ & $-^{f}$ & $-^{f}$ & \\
\hline \multirow[t]{2}{*}{500} & & $262 A \rightarrow G$ & Lys $88 \rightarrow$ Glu & $4 / 59$ & 2000 & $57 \pm 13$ & KO-1052 1055 \\
\hline & & ND & ND & $55 / 59$ & $--^{f}$ & $-^{f}$ & \\
\hline \multirow[t]{2}{*}{1000} & & $\begin{array}{l}\text { Insertion of } \\
\text { GGCGTGCGT } \\
\text { at } 250\end{array}$ & $\begin{array}{l}\text { Insertion of Gly, } \\
\text { Val, Arg at } 84\end{array}$ & $1 / 20$ & 3000 & $29 \pm 7$ & KO-1056 \\
\hline & & ND & ND & $19 / 20$ & $-{ }^{f}$ & $-^{f}$ & \\
\hline
\end{tabular}

Abbreviation: GYM, glucose-yeast extract-malt extract.

anumbered from the start codon of the open reading frame of $S$. griseus.

bDetermined after 5 days of incubation on GYM medium.

c $S$ trains were grown in SPY medium at $25^{\circ} \mathrm{C}$ for 3 days.

dWild-type strain.

eMutations were not detected in either rsmG or rpsL gene.

${ }^{f}$ Not determined.

reaction was carried out using a high-capacity RNA-to-cDNA Kit (Applied Biosystems, Foster City, CA, USA) according to the manufacturer's instructions. The samples were diluted with an appropriate volume of water and analyzed using the 7300 real-time quantitative PCR (qPCR) system and Power SYBR Green PCR master mix (Applied Biosystems). Each transcriptional assay was normalized to the corresponding transcriptional level of the $h r d B$ gene encoding the principal sigma factor. Primers used for real-time qPCR are listed in Table 1. All reactions were performed under the following conditions: $2 \mathrm{~min}$ at $50^{\circ} \mathrm{C}, 10 \mathrm{~min}$ at $95^{\circ} \mathrm{C}$, followed by 40 cycles of $15 \mathrm{~s}$ at $95^{\circ} \mathrm{C}$ for denaturing, $30 \mathrm{~s}$ at $60^{\circ} \mathrm{C}$ for annealing and $30 \mathrm{~s}$ at $72^{\circ} \mathrm{C}$ for extension.

\section{RESULTS AND DISCUSSION}

Isolation and characterization of streptomycin-resistant mutants In various actinomycetes, high-level resistance to streptomycin is often due to mutations in rpsL, which encodes the ribosomal protein S12, whereas low-level resistance is due to mutations in $r s m G$, which encodes a 16S rRNA methyltransferase.,6 Unlike other Streptomyces spp. (MIC to streptomycin, $0.2-2 \mu \mathrm{g} \mathrm{ml}^{-1}$ ), the streptomycin producer S. griseus IFO13189 showed a relatively high level of resistance $\left(\mathrm{MIC}=70 \mu \mathrm{g} \mathrm{ml}^{-1}\right)$. We isolated a total of 204 resistant mutant strains that had developed spontaneously on GYM agar plates containing various concentrations $\left(70-2000 \mu \mathrm{g} \mathrm{ml}^{-1}\right)$ of streptomycin. Of these mutants, 15 had mutations in the $r s m G$ gene and were characterized by the frequent appearance of deletion or insertion mutations that resulted in frameshift, and five carried mutations in $r p s L$, including the K88E mutation and novel insertion mutation (Table 2). These results were in contrast with those of previous studies in other Streptomyces spp, in which $>50 \%$ of resistant strains were due to mutations in $r p s L$ or $r s m G^{6}$ This observation suggested that $S$. griseus harbors a wide range of resistance systems, in addition to inactivation of streptomycin by streptomycin-6-phosphotransferase, to survive in the presence of its own antibiotic, streptomycin. The $r s m G$ mutants showed impaired ability to form aerial mycelia, and were somewhat deficient in sporulation.

We reported previously that, in the $r s m G$ mutant background, $r p s L$ mutants with high-level streptomycin resistance emerge at a frequency 200 -fold greater than that in the wild-type strain of S. coelicolor, and this elevated frequency in the emergence of high-level streptomycin resistance was facilitated by a mutation pattern in $\operatorname{rps} L$ more varied than that obtained by selection of the wild-type strain. ${ }^{5,6}$ Similarly, 
Table 3 Genes of Streptomyces griseus IF013189 analyzed in this study

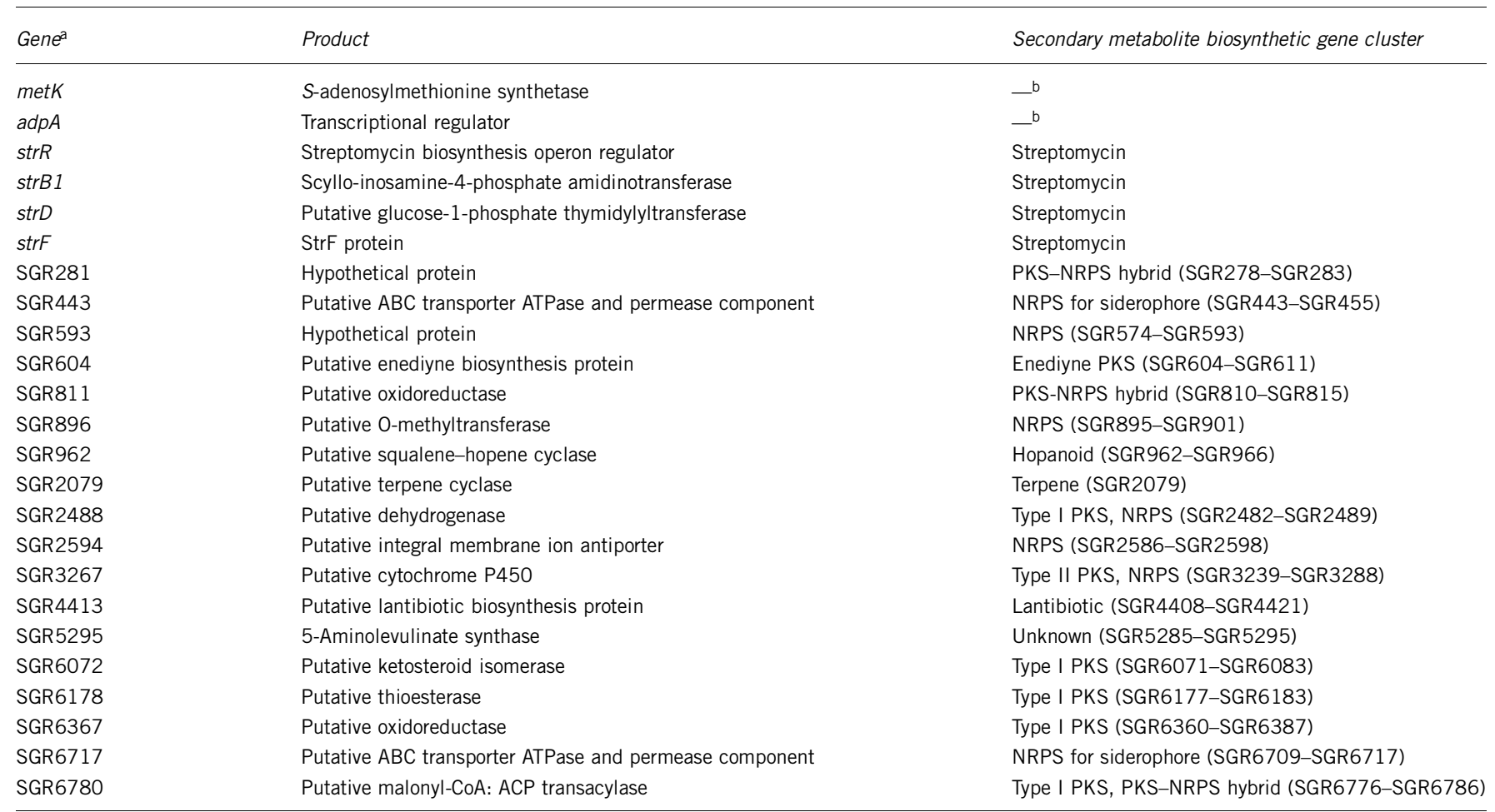

aGene names are obtained from the reference Ohnishi et al. ${ }^{4}$

bThese are not the secondary metabolite biosynthetic gene cluster.

S. griseus rsm $G$ mutant, KO-1050, generated the mutants resistant to high-level streptomycin $\left(1000 \mu \mathrm{g} \mathrm{ml}^{-1}\right)$ at high frequency. However, no $r p s L$ mutants were detected among the 30 resistant mutants tested, again characterizing the specific feature of $S$. griseus.

Effects of rsmG and rpsL mutations on streptomycin production Mutations in $r s m G$ were effective in enhancing streptomycin production, leading to a two- to threefold increase in streptomycin production, whereas $r p s L \mathrm{~K} 88 \mathrm{E}$ mutation and $r p s L$ insertion mutation were not effective (Table 2). In addition, the mutants with no mutations in either $r s m G$ or $r p s L$ (that is, ND mutants) showed the impaired ability to produce streptomycin, as examined with $12 \mathrm{ND}$ mutants (data not shown). In S. coelicolor, enhanced expression of the metK gene encoding $S$-adenosylmethionine synthetase corresponds to the enhanced production of streptomycin and actinorhodin caused by $r s m G$ mutations. ${ }^{5,12}$ Moreover, addition of $S$-adenosylmethionine causes overproduction of streptomycin in S. griseus, accompanied by enhanced transcription of $a d p A$ and $s t r R .{ }^{13,14}$ We analyzed transcription of $m e t K, a d p A$ and $s t r R$, together with several genes involved in biosynthesis of streptomycin ( $\operatorname{strB1}, \operatorname{strF}$ and $\operatorname{strD}$ ) (Table 3).

As expected, the $r s m G$ mutant, but not the $r p s L$ K88E mutant, exhibited elevated levels of metK, strR, strB1, strF and $s t r D$ expression compared with the wild-type strain at late growth phase $(36 \mathrm{~h})$ (Figure 1), thus underlying the enhanced production of streptomycin in the $r s m G$ mutant. StrR is a regulator of the streptomycin biosynthesis operon ${ }^{15}$ and AdpA acts as a central transcriptional regulator in the A-factor regulatory cascade of $S$. griseus. ${ }^{16}$ Although the elevated levels of metK and $s t r R$ expression account well for the marked enhancement of biosynthetic gene expression ( $\operatorname{strB1}$, strD and $s t r F)$, it is not yet clear why adpA gene expression was not activated in the $r s m G$ mutant.
Effects of rsmG mutation on transcription of secondary metabolitebiosynthetic gene clusters

A recent study in our laboratory indicated that certain mutations in $r p o B$ (encoding RNA polymerase $\beta$-subunit) or $r p s L$ genes can activate 'silent' genes of actinomycetes, leading to the discovery of novel antibacterial agents. ${ }^{17}$ The activation of silent genes by generating rpoB H437D or rpoB H437L mutations in Streptomyces sp. 631689 was attributed, at least partly, to the increased affinity of the mutant RNA polymerase for promoters. ${ }^{17}$ Thus, we analyzed the expression of secondary metabolite-biosynthetic gene clusters in the S. griseus rsmG mutant, KO-1050. A total of 18 genes belonging to 18 secondary metabolite-biosynthetic gene clusters (Table 3) were subjected to transcriptional analysis by real-time $\mathrm{qPCR}$, and the results indicated that the levels of expression of these genes are quite low $(1 / 10-1 / 1000$ compared with that of $\operatorname{str} B 1$ ), thus representing silent or poorly expressed genes. Strikingly, analysis of cells harvested at late growth phase $(36 \mathrm{~h})$ indicated that the $r s m G$ mutation affected not only streptomycin biosynthesis but also the expression of other secondary metabolite-biosynthetic genes, although the effects were not dramatic (Figure 2). Enhancement of expression was pronounced in SGR3267 (putative cytochrome P450 involved in Type II polyketide synthases or non-ribosomal peptide synthetases) and SGR962 (putative squalenehopene cyclase involved in hopanoid synthesis). By contrast, the $r p s L$ K88E mutant, KO-1052, did not show any enhanced expression of those genes (data not shown).

\section{CONCLUDING REMARKS}

We showed that $r s m G$ mutation is effective not only for enhancement of streptomycin production but also for activation of silent or poorly expressed genes in S. griseus. Although a biochemical approach (for example, measurement of in vitro protein synthesis activity) was not 

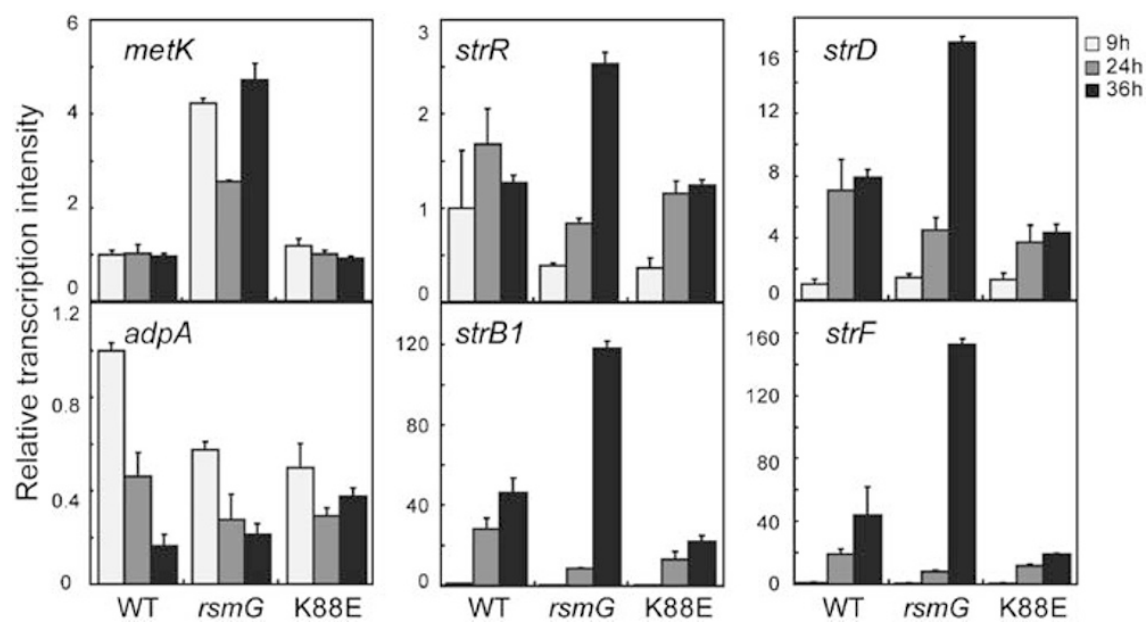

Figure 1 Transcriptional analysis of adpA, metK, strR, strB1, strD and $s t r F$ by real-time quantitative PCR (qPCR). The RNAs were extracted from cells of wild-type (13189), rsmG (KO-1050) and rpsL K88E (KO-1052) mutant strains grown to early growth phase (9h), mid growth phase (24h) or late growth phase $(36 \mathrm{~h})$ in SPY medium. Total RNA preparation and real-time qPCR were performed as described in Materials and Methods. The error bars indicate the standard deviations of the means of three or more samples.

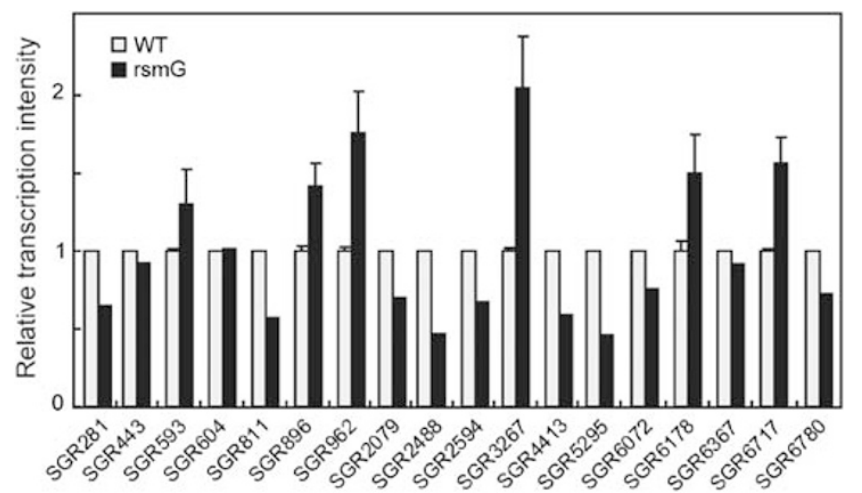

Figure 2 Transcriptional analysis of the genes involved in the secondary metabolite-biosynthetic gene clusters. The RNAs were extracted from cells of wild-type (13189) and rsmG (KO-1050) mutant strains grown to late growth phase $(36 \mathrm{~h})$. Total RNA preparation and real-time quantitative PCR was performed as described in Materials and Methods. Error bars indicate the standard deviations of the means of three or more samples.

employed in the present study, it is possible that the expression of pathway-specific regulatory genes is governed by higher-order regulatory proteins and expression of such higher-order regulatory proteins may be significantly affected under conditions associated with enhanced metK expression in mutants. The present method, together with other methods reported recently, ${ }^{6,7,17-20}$ may be useful for activating silent genes, eventually leading to the discovery of novel antibacterial agents.

\section{ACKNOWLEDGEMENTS}

This work was supported by grants to KO (Effective Promotion of Joint Research of Special Coordination Funds) from the Ministry of Education, Culture, Sports and Technology of the Japanese Government.

\section{CONFLICT OF INTEREST}

The authors declare no conflict of interest.

1 Newman, D. J. \& Cragg, G. M. Natural products as sources of new drugs over the last 25 years. J. Nat. Prod. 70, 461-477 (2007).
2 Bentley, S. D. et al. Complete genome sequence of the model actinomycete Streptomyces coelicolor A3(2). Nature 417, 141-147 (2002).

3 Ikeda, H. et al. Complete genome sequence and comparative analysis of the industrial microorganism Streptomyces avermitilis. Nat. Biotechnol. 21, 526-531 (2003).

4 Ohnishi, Y. et al. Genome sequence of the streptomycin-producing microorganism Streptomyces griseus IFO 13350. J. Bacteriol. 190, 4050-4060 (2008).

5 Nishimura, K., Hosaka, T., Tokuyama, S., Okamoto, S. \& Ochi, K. Mutations in rsmG, encoding a 16S rRNA methyltransferase, result in low-level streptomycin resistance and antibiotic overproduction in Streptomyces coelicolor A3(2). J. Bacteriol. 189, 3876-3883 (2007).

6 Tanaka, Y. et al. Antibiotic overproduction by $r p s L$ and $r s m G$ mutants of various actinomycetes. Appl. Environ. Microbiol. 75, 4919-4922 (2009).

7 Wang, G., Hosaka, T. \& Ochi, K. Dramatic activation of antibiotic production in Streptomyces coelicolor by cumulative drug resistance mutations. Appl. Environ. Microbiol. 74, 2834-2840 (2008).

8 Ochi, K. From microbial differentiation to ribosome engineering. Biosci. Biotechnol. Biochem. 71, 1373-1386 (2007).

9 Tamehiro, N. et al. Innovative approach for improvement of an antibiotic-overproducing industrial strain of Streptomyces albus. Appl. Environ. Microbiol. 69, 6412-6417 (2003).

10 Beltrametti, F., Rossi, R., Selva, E. \& Marinelli, F. Antibiotic production improvement in the rare actinomycete Planobispora rosea by selection of mutants resistant to the aminoglycosides streptomycin and gentamycin and to rifamycin. J. Ind. Microbiol. Biotechnol. 33, 283-288 (2006).

11 Ochi, K. Metabolic initiation of differentiation and secondary metabolism by Streptomyces griseus: significance of the stringent response (ppGpp) and GTP content in relation to A factor. J. Bacteriol. 169, 3608-3616 (1987).

12 Okamoto, S., Lezhava, A., Hosaka, T., Okamoto Hosoya, Y. \& Ochi, K. Enhanced expression of S-adenosylmethionine synthetase causes overproduction of actinorhodin in Streptomyces coelicolor A3(2). J. Bacteriol. 185, 601-609 (2003).

13 Saito, N., Kurosawa, K., Xu, J., Okamoto, S. \& Ochi, K. Effect of S-adenosylmethionine on antibiotic production in Streptomyces griseus and Streptomyces griseoflavus. Actinomycetologica 17, 47-49 (2003).

14 Shin, S. K., Xu, D., Kwon, H. J. \& Suh, J. W. S-adenosylmethionine activates adpA transcription and promotes streptomycin biosynthesis in Streptomyces griseus. FEMS Microbiol. Lett. 259, 53-59 (2006).

15 Retzlaff, L. \& Distler, J. The regulator of streptomycin gene expression, StrR, of Streptomyces griseus is a DNA binding activator protein with multiple recognition sites. Mol. Microbiol. 18, 151-162 (1995).

16 Ohnishi, Y., Yamazaki, H., Kato, J. Y., Tomono, A. \& Horinouchi, S. AdpA, a central transcriptional regulator in the A-factor regulatory cascade that leads to morphological development and secondary metabolism in Streptomyces griseus. Biosci. Biotechnol. Biochem. 69, 431-439 (2005).

17 Hosaka, T. et al. Antibacterial discovery in actinomycetes strains with mutations in RNA polymerase or ribosomal protein S12. Nat. Biotechnol. 27, 462-464 (2009).

18 Rigali, S. et al. Feast or famine: the global regulator DasR links nutrient stress to antibiotic production by Streptomyces. EMBO Rep. 9, 670-675 (2008).

19 Carata, E. et al. Phenotypes and gene expression profiles of Saccharopolyspora erythraea rifampicin-resistant (rif) mutants affected in erythromycin production. Microb. Cell. Fact. 8, 18 (2009).

20 Tala, A. et al. Activation of dormant bacterial genes by Nonomuraea sp. strain ATCC 39727 mutant-type RNA polymerase. J. Bacteriol. 191, 805-814 (2009). 